\title{
Kirche auf dem privaten Radiomarkt Wie sind Verkündigung und kirchliche Themen im Kommerzfunk durch die Landesrundfunkgesetze geregelt?
}

\author{
von Waldemar Schmid*
}

Am 12.11.1984 erhielt der damalige Vorsitzende der Länder-Rundfunkkommission und rheinland-pfälzische Ministerpräsident, Bernhard Vogel, ökumenische Post: Peter Düsterfeld (damals Leiter der Zentralstelle Medien der Deutschen Bischofskonferenz) und Hans-Wolfgang Heßler (damals Leiter des Gemeinschaftswerkes der evangelischen Publizistik) äußerten in einem gemeinsamen Brief Gedanken zum Entwurf eines Länder-Staatsvertrages. Dieser „Staatsvertrag zur Neuordnung des Rundfunkwesens" sollte bundesweit den Überbau über bestimmte Regelungsbereiche der Länder-Rundfunkgesetzgebung bilden, die im Übergang zum Nebeneinander von öffentlich-rechtlichem und privatem Rundfunk war. Unter Punkt 5 des ökumenischen Schreibens hieß es: „Die Kirchen erwarten aufgrund ihrer qualifizierten Position im Rundfunk auch in den neuen Rundfunkprogrammen angemessene Berücksichtigung“. Weiter unten betonten die beiden Absender: „Die Kirchen bitten Sie dringend um eine gesetzliche Regelung durch den Staatsvertrag in diesem Sinne, weil eine (konkurrierende) Präsenz der Kirchen im öffentlich-rechtlichen oder im privatrechtlichen Rundfunk jeweils zu ungleichen Bedingungen, zu schweren innerkirchlichen Problemen führen würde und letztlich auch fatale gesellschaftliche Folgen hätte."

Das war klug und fürsorglich geschrieben, besonders auch für die kirchliche Binnensicht, denn intern grummelte es: Das anstehende finanzielle Engagement der Ortskirchen im privaten Rundfunk (Hörfunk und Fernsehen) drohte u. a. die Stellung der katholischen Senderbeauftragten für die öffentlich-rechtlichen Anstalten zu erschweren und das sensible Regelwerk der „Verkündigungssendungen“ zu gefährden. Hier mußten sich die Ortskirchen in die Pflicht des Augenmaßes nehmen lassen. Etwas gut zwei Jahre später, am 26.01.1987, forderten die genannten Senderbeauftragten in einer Erklärung unter anderem, daß das PrivatfunkEngagement der Ortskirchen vom Umfang her die Aufwendungen bei den öffentlich-rechtlichen Anstalten nicht übersteigen sollte. Das bestätigten die katholischen Bischöfe bei ihrer Frühjahrsvollversammlung 1987.

Möglich gemacht wurde die „duale“ Rundfunklandschaft durch das sog. „FRAG“-Urteil des Bundesverfassungsgerichts von 1981, das dritte höchstrichterliche Fernsehurteil aus Karlsruhe: Die Verfassungshüter hielten privaten Rundfunk für erlaubt, wenn freie und umfassende Meinungsbildung möglich sei, und dazu sollte das Zuwortkommen der gesellschaftlichen Kräfte im Gesamtprogramm künftiger privater Rundfunkanbieter gehören. Es verstand sich, daß sich die Kirchen ganz wesentlich mit zu diesen gesellschaftlichen Kräften zählten.

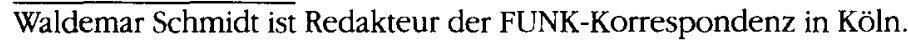


Einen Monat vor der geplanten Unterzeichnung dieses Staatsvertrages, am 04.03.1987, schrieben die beiden Kirchen wiederum gemeinsam einen Brief, diesmal an alle Ministerpräsidenten als vorgesehene Unterzeichner des Vertragswerkes: Eine „Kann-Vorschrift“ über die Berücksichtigung kirchlicher Sendungen im Privatrundfunk bedeute eine Nichtbeachtung der besonderen Stellung der Kirchen in der Gesellschaft, hieß es dort mahnend.

Diese Mahnung signalisiert: Es wurde noch bis zuletzt auch darum gerungen, ob und auf welche Weise kirchliche Präsenz im privaten Rundfunk (Hörfunk und Fernsehen) verbindlich festgelegt werden sollte. Anders als der gebührenfinanzierte öffentlich-rechtliche Rundfunk muß sich privater Rundfunk ausschließlich durch Werbeeinnahmen und damit über Hörerzahlen finanzieren. Der Programmteil „Kirche“, der dort als selbstverständlicher Teil der Grundversorgung betrachtet wurde, konnte hier zum Problemfall werden: Wie konnten Religion und Kirche in Programmen vertreten sein, die ganz elementar den Wandel der Gesellschaft zur restlosen Kommerzialisierung auch der Kommunikation repräsentieren würden (und bereits repräsentierten), und die gleichzeitig den Wandel zur restlosen Säkularisierung signalisierten. Ohne mediengesetzliche Rückendeckung wäre es den Kirchen schwer gefallen, ihre formalen Ansprüche in der Gesellschaft der 80er Jahre gegenüber dem Kommerzrundfunk durchzusetzen. Der Schlüssel war der Spruch der Karlsruher Richter.

Die Lobbytätigkeit der beiden großen Kirchen im unmittelbaren Vorfeld des Rundfunk-Staatsvertrages von 1987 war nur ein Aspekt ihrer medienpolitischen Arbeit. Die Frage nach dem „Plansoll“ kirchlicher Verkündigung und kirchlich relevanter Berichtsthemen im bundesdeutschen Rundfunk stellte sich in den 80er Jahren anders dar als Ende der 40er Jahre, als es die ARD noch nicht und das ZDF noch lange nicht gab. Die Präsenz von Kirche in den Programmen von ARD (Hörfunk und Fernsehen) und ZDF wurde in den 80er Jahren, was die Quantitäten anbelangt, als angemessen erachtet. Als kommerzieller Rundfunk vor der Tür stand, mußten vor der Folie nicht nur des schon Bestehenden, sondern auch einer neuen Gesellschaft, nämlich jener der 80er Jahre und der sie mit repräsentierenden kommerziellen Programme, neue Kriterien des gesellschaftlich „Notwendigen" bezüglich religiöser Sendungen im kommerziellen Rundfunk definiert werden.

Der neue Staatsvertrag enthielt schließlich, wenn auch verschlüsselt konditional, in Art. 8, Abs. 1 eine Muß-Vorschrift: „Die bedeutsamen politischen, weltanschaulichen und gesellschaftlichen Kräfte müssen in den Vollprogrammen angemessen zu Wort kommen“. Seither wird „angemessen“ faktisch übersetzt mit „vergleichbar den öffentlich-rechtlichen Programmen“, wenn auch Gottesdienstübertragungen im kommerziellen Hörfunkbereich noch nie stattfanden, im Fernsehbereich mittlerweile dreimal (RTLplus in der Osternacht). Die formale Stellung der Kirchen im Rundfunk-Staatsvertrag wurde von den deutschen Bischöfen auf ihrer Herbst-Vollversammlung 1987 ausdrücklich begrüßt, außerdem wurde dort betont, daß das Privatrundfunk-Engagement der Einzeldiözese vor dem überdiözesanen Engagement rangieren sollte; und schließlich wurde festgestellt, daß der 1987 gegründete "Verein Katholischer Rundfunk" eine private Initiative sei, keine bischöflich getragene. Damit wurde signalisiert, daß sich die katholischen Bistümer in Deutschland keinen eigenen privaten kirchlichen Hörfunksender leisten 
wollten. Die informelle Organisation der kirchlichen Senderbeauftragten für den Privatfunk, KAPRI genannt (Katholische Kirche im Privatfunk), wurde von den Bischöfen im Herbst 1987 bestätigt.

\section{Gesetzesinitiativen mußten harmonisiert werden}

Ende 1984 waren in den damals noch elf Bundesländern fünf Landesrundfunkgesetze in Vorbereitung, außerdem drei Versuchsgesetze für „Breitbandkabelversuche" bereits erlassen, das Berliner Kabelgesetz ebenfalls noch in Vorbereitung. Im Norden der Bundesrepublik stand 1984 die Lizenzierung von landesweit ausgestrahltem Radio an, zunächst in Niedersachsen, dann auch in SchleswigHolstein. In Bayern und Baden-Württemberg wurden seit 1984 lokale und regionale Sendestationen geplant. Wo Lokalfunk geplant war, ergab sich die Möglichkeit, daß Kirche auf lokaler Ebene, im „Nahraum“, elektronisch präsent sein konnte. Ein neues Kommunikationskonzept, das teilweise, nicht überall, mit Euphorie begrüßt wurde.

Die Lage in den verschiedenen Regelungsbereichen (die Frage der Präsenz der Kirchen in den Programmen war lediglich eine unter vielen) war uneinheitlich. Es ging den Bundesländern mit dem geplanten Staatsvertrag um die größtmögliche Harmonisierung der bisher verabschiedeten Landesmediengesetze bzw. Gesetzentwürfe und nicht zuletzt um Regelungen des Satellitendirektempfangs, der mittlerweile drohte, die Länderhoheit im Rundfunkwesen zur lächerlichen Petitesse werden zu lassen.

Entsprechend den Landesmediengesetzen stehen den Kirchen „angemessene" Sendezeiten für religiöse Sendungen auch in den Privatprogrammen verbindlich zu. Das entspricht der schließlich im Rundfunkstaatsvertrag von 1987 gemachten Vorgabe. Für die Kirchen ergab sich in der Landesrundfunkgesetzgebung zudem, daß sie als gesellschaftlich relevante "Gruppen" meist mit an erster Stelle ihre Vertreter in die Lizenzierungs- und Programmaufsichtsgremien der jeweiligen „Landesmedienanstalten / Landesanstalten für das Rundfunkwesen / für die neuen Medien“ entsenden durften. Diese neuen Körperschaften sind öffentlich-rechtlich und werden durch einen 20-Pfennig-Zuschlag zur Rundfunkgebühr finanziert.

Es entstanden somit viele neue Gremienmitgliedschaften. Denn nicht nur in die "großen" Gremien der Landesmedienanstalten (neben Rundfunkräten bzw. Fernsehrat von ARD und ZDF sowie Deutsche Welle undDLF, demnächst Deutschlandradio) können Kirchenvertreter entsandt werden, sondern auch in die kleinen bis winzigen Versammlungen, etwa der bayerischen lokalen „Kabelgesellschaften“ oder der "Veranstaltergemeinschaften“ der Lokalsender in Nordrhein-Westfalen.

\section{Die „Drittsenderechte}

Die Kann-Bestimmung des „Selbstkostenersatzes“ für die Ausstrahlung kirchlicher Beiträge in kommerziellen Hörfunk- und Fernsehprogrammen ist in den meisten Landesrundfunkgesetzen unter den Paragraphen „Beiträge Dritter“ (mittlerweile auch im neuen baden-württembergischen Landesrundfunkgesetz 
und in den Landesrundfunkgesetzen der neuen Bundesländer) gesetzlich festgelegt. Sie war u. a. auch ein Anlaß für die eingangs erwähnte Mahnung von Düsterfeld/Heßler an den damaligen rheinland-pfälzischen Ministerpräsidenten und Katholiken Bernhard Vogel. Sie hatte - neben dem Kostenfaktor an sich - auch eine wichtige medienpolitische Komponente: Wie sollten die Kirchen gegenüber ARD und ZDF die in Jahren entstandene Gewohnheit weiter verteidigen? Dort werden Gottesdienstübertragungen unter kirchlicher Verantwortung, aber auf Kosten der Anstalten gesendet, außerdem Verkündigungssendungen (Kurzpredigten, Morgenandachten) von den Anstalten honoriert, schließlich unterhalten die öffentlichrechtlichen Anstalten auf eigene Kosten „Kirchenfunk-Redaktionen“ (deren redaktionelle Trends den Kirchen freilich nicht immer genehm waren, so daß sie den Privatfunk mit der Möglichkeit, kirchliche Beiträge unter eigener Regie zu produzieren, sogar als willkommene Möglichkeit betrachteten).

Das Problem wurde dadurch entschärft, daß Gottesdienstübertragungen in privaten Hörfunk völlig unterblieben, daß es außerdem im Privatfernsehen mit drei bisherigen Ausnahmen (von RTLplus übertragene Osternachtfeiern aus Taizé und Fiona) ebenfalls keine Gottesübertragungen gab. Außerdem wurde die Praxis der Selbstproduktion von Verkündigungs- und redaktionellen Sendungen durch kircheneigene bzw. kirchennahe Produktionsstudios entwickelt. Die bislang übliche Finanzregelung zwischen Privatstationen und Kirchen ist die, daß pragmatisch Sende-Selbstkosten mit möglichen Honoraren aufgerechnet werden, denn in den meisten Individualverträgen ist festgelegt, daß dann, wenn die Sender in die schwarzen Zahlen kommen, Honorare für die Zulieferungen fällig sind. Der wichtigste Faktor bei der Entschärfung der Frage des „Selbstkostenersatzes“ war und ist aber der, daß die Kirchen bei vielen privaten Hörfunkveranstaltern Mitgesellschafter sind, meist über überdiözesane kirchliche Firmen.

\section{Programmpräsenz: Kirche geht auch zur Disco}

Eine weitere Problematik ergab sich aus programminhaltlichen Erwägungen: In einer neuen Radiokultur mußte, wie der Bereich des kommerziellen Hörfunks bereits damals gezeigt hatte, die Darstellung von "Kirche" notwendigerweise anders aussehen als im gewohnten öffentlich-rechtlichen Programmumfeld. Aber - würde dieses andere Aussehen noch „kirchlich“ sein? Für eine relativ junge Hörerschaft bestimmt, nämlich zunächst vorrangig für die 16- bis 36-jährigen, erforderte das schrille Hörmilieu vieler Privatsender gänzlich neue kirchliche Instrumente der Ansprache. Wo bislang etwa im SWF 3-Milieu ein eingestreuter (floatender) kirchlicher Verkündigungsbeitrag als Beinahe-Fremdkörper betrachtet werden mußte, da wurde das im Privathörfunkmilieu zum Kirchenalltag schlechthin. Die Frage des Gelingens einer ins Format passenden „kirchlichen Sendung“ wurde zur Überlebensfrage für die Sender, die keine Durchhänger dulden konnten - und damit zur Qualitätsfrage der Zusammenarbeit zwischen Sender und Kirche.

Innerkirchlich wichtiger war die Frage nach den Konzepten kirchlicher Ansprache jener anderen, neuen Hörer der neuen privaten Hörfunkprogramme, damit nach der "Reichweite“ jedes Sprechens von Kirche und von christlicher Botschaft vor einer als bestenfalls desinteressiert vermuteten Hörerschaft. Es brauchte 
neben unkonventionellen Ansätzen vor allem einen neuen, jungen Typus von kreativem Potential in diesem Sektor kirchlicher Tätigkeit.

Zur Frage der Programmpräsenz entwickelten sich je nach Bundesland und nach Mentalität unterschiedliche Formen, die mit den neuen medienrechtlichen Regelungen zunächst wenig zu tun hatten. Erstaunlich dabei: Die Quantität kirchlicher Präsenz wurde im Privathörfunksektor im Vergleich zu den ARD-Hörfunkprogrammen nicht geringer. Sie war, und das ist im Rahmen der sich sehr „jugendgemäß" und meist säkular gebenden neuen Hörfunkprogramme aber nur vordergründig erstaunlich, sogar erwünscht. Aus einsichtigen Gründen: Die Kirchen als Selbstproduzenten von Beiträgen waren zunächst (und zum Teil nach wie vor) für die Sender ein willkommener Kostensenkungsfaktor. Allein dies zu sehen wäre allerdings zu kurz gegriffen, weil die personelle Interaktion zwischen den Redaktionen der Sender und den Kirchenredaktionen sich in den meisten Fällen gut entwickelte und somit zum positiven Berührungsfaktor jenseits von Ideologieoder Kommerzüberlegungen wurde. Die fachliche Anerkennung kam schließlich hinzu.

Im Fernsehbereich blieben die „kirchlichen Quantitäten“ zunächst außerordentlich gering, beschränkten sich bei RTLplus zunächst auf Fünfminuten-Spots pro Woche wie "Bild als Botschaft“ und bei SAT 1 „So gesehen“.

\section{Einige Länderbeispiele}

\section{Bayern:}

Seit dem 16.07.1982 gab es in Bayern einen „Grundvertrag für das Kabelpilotprojekt München“, die Urzelle des späteren (und jetzt abgelösten) bayerischen „Medienentwicklungs- und Erprobungsgesetzes (MEG)“. Besondere Regelungen für Gremienvertretung, Erlaubnisvoraussetzungen und Sonder-Sendezeiten für die Kirchen gab es dort noch nicht, wohl aber im MEG vom 15.11.1984. Art. $111 \mathrm{der}$ bayerischen Verfassung besagt auch, daß Rundfunk in Bayern nur unter öffentlichrechtlicher Verantwortung stattzufinden habe. Deshalb wurde dort die öffentlichrechtlich verfaßte Lizenzierungs- und Programmverantwortungsbehörde "Bayerische Landesanstalt für neue Medien“ eingerichtet. In deren „Medienrat" sind die Kirchen mit dabei (Art. 12): je ein Vertreter der katholischen und evangelischen Kirche sowie der jüdischen Kultusgemeinde.

Grundlage für die Präsenz von kirchlichen/religiösen Sendungen im Privatfunk Bayerns ist Art. 25 des MEG („Beteiligung der Anbieter"). Religions- oder Weltanschauungsgemeinschaften können den Kabelgesellschaften Rundfunkprogramme und -sendungen anbieten. Die sieben bayerischen Diözesen sind weder an der bayernweit tätigen "Antenne Bayern" noch an der neuen bayernweiten „Bayerische Lokal-Radio-Programm GmbH“ (BLR) finanziell direkt beteiligt; die Beteiligungen am (lokalen) Privatfunk geschehen meist indirekt über kirchliche Vereine bzw. Körperschaften. 


\section{Baden-Württemberg:}

Von Erzbischof Oskar Saier (Freiburg) und dem inzwischen verstorbenen Bischof Georg Moser (Rottenburg-Stuttgart), der seinerzeit der Publizistischen Kommission der Deutschen Bischofskonferenz vorsaß, stammt die gemeinsame Stellungnahme vom 27.06.1983 zum Landesmediengesetzentwurf von BadenWürttemberg. Saier und Moser sprachen sich in diesem an Lothar Späth adressierten Papier eindeutig für die binnenplurale und öffentlich-rechtliche Organisation von Rundfunk aus. Wörtlich schrieben sie u. a.: „Es muß demnach die Gefahr ausgeschlossen werden, daß Informationen und Meinungen, die von allgemeinem Interesse sind, von der öffentlichen Meinungsbildung ausgeschlossen werden und jene Meinungsträger, die sich im Besitz von Sendefrequenzen und ausreichenden Finanzmitteln befinden, die öffentliche Meinungsbildung vorherrschend bestimmen ..." Es sollte, wie es weiter hieß, „zumindest eine hinreichende Wahrscheinlichkeit bestehen, daß sich eine angemessene Vielfalt einstellt". Ein Hauptansatzpunkt der Kritik war der, daß im Gesetzentwurf vom Juli 1983 dem Axiom der Gleichgewichtigkeit der Kräfte Vorrang gegeben wurde, ohne im einzelnen darauf einzugehen, daß die gesellschaftlichen Gruppen nur sehr unterschiedlich stark in der Lage sind, sich Zugang zu den Kommunikationsmitteln zu verschaffen.

Grundlage für die Präsenz der Kirchen im baden-württembergischen Privatrundfunk waren zunächst die Artikel 18 und 44 des baden-württembergischen Landesmediengesetzes vom 12.12.1985. Nach Art. 18 ("Vielfaltsicherung") gab die neugegründete „Landesanstalt für Kommunikation“ (LfK) den Lizenzbewerbern verbindlich vor, die Grundsätze der Meinungsvielfalt (Art. 14, Abs. 2, hier Satz 2) dadurch zu realisieren, daß sie den Kirchen Sendezeiten einräumten und einen entsprechenden Vertrag vorwiesen. 90 Minuten pro Woche und Programm war die Regelzeit für die beiden Kirchen (evangelisch und katholisch) gemeinsam, die sie dann untereinander aufteilten, normalerweise hälftig.

Die beiden katholischen Bistümer in Baden-Württemberg beteiligten sich nirgendwo selbst an einer Trägerfirma für eine private Radiostation oder einem privaten Fernsehsender. Zunächst hatten beide Kirchen vorsorglich eigene Hörfunk-Lizenzanträge für (kirchliche) Spartenprogramme für jede der ausgeschriebenen Hörfunkfrequenzen gestellt. Tatsächlich hat aber nie eine Kirche in BadenWürttemberg eine Spartenprogramm-Sendelizenz erhalten, auch keine benötigt, weil durch die Praxis der Lizenzerteilung den Kirchen weitgehende Sendemöglichkeiten eingeräumt worden waren.

Diese Sendemöglichkeiten wurden im neuen Landesmediengesetz BadenWürttemberg vom 17.03.1992 in ein förmliches "Drittsenderecht" umgewandelt (Art. 62), allerdings nur in Vollprogrammen. Die Religionsgemeinschaften (römisch-katholische Kirche, evangelische Landeskirchen, jüdische Religionsgemeinschaft) sind somit faktisch den Bundesbehörden und dem Katastrophendienst gleichgestellt; die Veranstalter können die Erstattung ihrer Selbstkosten verlangen.

Die konkrete Kirchenfunk-Tätigkeit wurde von den beiden Diözesen im Südweststaat sehr unterschiedlich organisiert: Während die Erzdiözese Freiburg ein eigenes „Institut für Medienarbeit" mit einem Produktionsstudio in Freiburg 
einrichtete, übergab die Diözese Rottenburg-Stuttgart die Redaktion und Produktion der Beiträge für die Sender in ihrem Gebiet dem APG-Unternehmen „Kirche im privaten Rundfunk (KiP)" in Stuttgart. Die APG ist eine Tellux-Tochter, die wiederum mehreren deutschen Diözesen gehört. "KiP" ist mittlerweile nicht nur in Baden-Württemberg tätig, sondern auch in Nordrhein-Westfalen („KiP-NRW“) sowie in Sachsen.

Die Erzdiözese Freiburg unterhält in Freiburg ein bistumseigenes Produktionsstudio für Verkündigungs- und journalistische Beiträge. Wesentlich für den badischen (Freiburger) Bereich ist, daß viel mit festen freien Mitarbeitern gearbeitet wird, die in der Regel ein Fixum plus Einzelhonorare bekommen; für den württembergischen Bereich (Rottenburg-Stuttgart) ist wesentlich, daß es ausschließlich Festangestellte sind, die teils von der Diözese, teils von der APG bezahlt werden. Jeder Redakteur betreut, wie im Freiburger Bereich, „seine" Sender redaktionell und ist zugleich kirchlicher Senderbeauftragter für seinen Sender.

\section{Niedersachsen:}

Kurz vor der Verabschiedung des niedersächsischen Landesrundfunkgesetzes vom 23.05.1984 beschlossen die Bistümer Hildesheim und Osnabrück sowie das Offizialat Vechta (Bistum Münster), in einem kommenden niedersächsischen Privat-Hörfunkprogramm gemeinsam ein "katholisches Fenster" zu finanzieren. Federführend bei diesem Projekt sollte das Bistum Hildesheim sein. Der Hildesheimer Bernward-Verlag, der zu $90 \%$ im Besitz der Diözese Hildesheim ist, wurde mit dem Aufbau eines Produktionsstudios beauftragt. Der Bernward-Verlag seinerseits beteiligte sich zusammen mit dem Osnabrücker Kirchenbote-Verlag im Umfang von jeweils $102.000 \mathrm{DM}$ an der "Mediengesellschaft Niedersachsen mbH" (30 Verlage), die mit ihrerseits $13 \%$ an der „Funk \& Fernsehen Nordwestdeutschland GmbH \& Co KG" beteiligt ist, die das landesweit sendende „Radio ffn“ veranstaltet. Am mittlerweile zweiten landesweiten Hörfunksender, „Antenne Niedersachsen“ (Radio Gong Niedersachsen), sind auf katholischer Seite die Bernward-Tochter Calig GmbH und die Dombücherstube Osnabrück mit jeweils 75.000 DM finanziell direkt beteiligt. Mit insgesamt 150.000 DM ist an Antenne Niedersachsen auch die evangelische Kirche über die Gründung „Evangelischer Kirchenfunk Niedersachsen (ekn)" in Hannover (acht Gesellschafter aus dem evangelischen Bereich) beteiligt. Die Beteiligung von unterschiedlichen, wenngleich miteinander verflochtenen Unternehmen entspricht dem Buchstaben des niedersächsischen Landesrundfunkgesetzes, das einem Veranstalter jeweils ein Vollprogramm und ein sonstiges Programm (Spartenprogramm) zubilligt. Beide privaten Hörfunkprogramme gelten als Vollprogramme (Information, Bildung, Beratung, Unterhaltung).

Im 26köpfigen niedersächsischen Landesrundfunkausschuß sind die Kirchen mit jeweils einem Mitglied vertreten (Art. 24, Abs. 1 Landesrundfunkgesetz). Entsprechend Art. 25, Satz 3 darf ein Programmveranstalter aber nicht Mitglied im Landesrundfunkausschuß sein. Dem wird durch die Konstruktion der lediglich kirchennahen, indirekten Beteiligung an den Programmveranstaltern entsprochen. Unabhängig vom Veranstalterstatus werden den Kirchen besondere Sendezeiten (Drittsenderecht) zugestanden, mit der Kann-Vorschrift der Selbstkostenerstattung. 
Im Entwurf vom Januar 1993 für das neue niedersächsische Landesrundfunkgesetz hat sich in Art. 34 eine Forderung der Grünen nach der Zulassung von nichtkommerziellem lokalem Hörfunk niedergeschlagen. Das hat bereits im Vorfeld der Verabschiedung des Gesetzes zur Entschärfung eines Rechtsstreits beigetragen, der seinerzeit als Fußnote durch den Blätterwald ging: Der katholische Pastor Jan van den Brule hatte mit technischen und jurististischen Kniffs die illegale Hörfunkausstrahlung von Sonntagsgottesdiensten in seiner Gemeinde „Mariä Verkündigung" in Duderstadt-Breitenberg bewerkstelligt. Im Vorfeld der Gesetzesnovellierung hatte sich auch eine „Interessengemeinschaft Gemeinnütziger Hörfunk Niedersachsen (INGEHN)“ gebildet, der auch der „Kirchengemeindefunk Breitenberg“ angehört. Nun sollen also „Betriebsversuche zur Veranstaltung von lokalem nicht kommerziellem Hörfunk" möglich gemacht werden. Diese Versuche sollen dann starten, wenn „mindestens zwei Modellprojekte den Sendebetrieb aufgenommen haben", fünf Jahre dauern und wissenschaftlich begleitet werden.

\section{Rheinland-Pfalz:}

Am 01.01.1984 fand der aufwendig bejubelte Privatfunk-"Urknall" beim Ludwigshafener Kabelpilotprojekt statt. Ein Vierteljahr zuvor am 01.10.1983 hatte das Bistum Mainz eine Abteilung „Kirche und Medien“ eingerichtet. Die sollte zwar zunächst die rundfunkpolitische Entwicklung in Rheinland-Pfalz beobachten, baute aber bald in ihren Räumen ein kleines Hörfunk-Produktionsstudio auf. Im Rahmen des rheinland-pfälzischen Breitband-Versuchsgesetzes beantragte das Bistum Mainz formell eine eigene Nutzungsgenehmigung, gedacht war aber an Kooperationen. Die Chance hierfür tat sich auf, als in Mainz die gemeinnützige „Landeskooperationsgemeinschaft Bürgerservice Hörfunk" gegründet wurde. Daran beteiligte sich das Bistum. Der „Bürgerservice“ verhandelte mit der „Rheinland-Pfälzischen Rundfunk-Gesellschaft GmbH“ (RPR) über Sendezeiten. Die (indirekte) Mitgliedschaft der katholischen Kirche bei RPR verstärkte deren gesellschaftliche Relevanz, RPR erhielt schließlich als Lizenzbewerber zunächst $72 \%$ der Sendezeit auf der ersten UKW-Privathörfunkkette. Mittlerweile besitzt die RPR die gesamte Sendezeit und wurde auch für die zweite Privathörfunkkette lizenziert.

Das Bistum Trier hat im Unterschied zum Bistum Mainz nicht selbst investiert, sondern den zu $90 \%$ bistumseigenen Paulinus-Verlag mit der Kirchenfunkproduktion beauftragt. Paulinus ist mit einem kleinen Anteil Gründungsgesellschafter an der Rheinland-Pfälzischen Rundfunk-Gesellschaft (RPR), war zunächst auch an den selbständigen Regionalstudios beteiligt. Nachdem die Studios zu unselbständigen RPR-Studios wurden, nahm Paulinus seine Anteile zurück.

Die Kirchenfunkredakteure der beiden RPR-Regionalstudios im Trierer Bereich stehen auf der Paulinus-Gehaltsliste. Sie arbeiten zwar bei RPR mit den Schwerpunkten Kultur und Kirche; produzieren aber auch andere, nichtkirchliche journalistische Beiträge.

Bei der Leitung von Paulinus vermied man im Privathörfunk-Engagement das Genre „Verkündigungssendungen“ im strengen Sinne. Vielmehr soll "Kirche“ im Privatfunk ausschließlich durch journalistische Beiträge präsent sein; die sich mit Vorgängen im Umkreis von Kirche befassen. Dieses Konzept entspricht der Verlagsphilosophie in anderen Medienengagements des Unternehmens. Man ist 
bei Paulinus der Ansicht, daß das „Drittsendungsrecht" des Auftraggebers Diözese Trier mit diesem Konzept legitim wahrgenommen wird.

Im Bereich des Bistums Speyer war für die Verkündigungssendungen, die im RPR-Studio Ludwigshafen ausgestrahlt wurden, zunächst eine „Autorengruppe Pastoraltheologie" unter der Aufsicht von Domkapitular Johannes Dörr eingerichtet worden. Mittlerweile hat auch Speyer einen eigenen Redakteur für die Privathörfunksender im Diözesanbereich, der zugleich wie in Mainz kirchlicher Privathörfunkbeauftragter ist.

Schon im rheinland-pfälzischen "Landesgesetz für einen Versuch mit Breitbandkabel" vom 04.12.1980 waren die Kirchen als Mitglieder der "Versammlung" genannt (Art. 8, Abs. 1). Im nachfolgenden Landesrundfunkgesetz vom 24.06.1986 wie auch in der nachfolgenden Novelle von 1988 sowie im neuen Landesrundfunkgesetz vom 16.07.1992 gehören die katholischen Bistümer in Rheinland-Pfalz zu denjenigen Körperschaften, die in der Versammlung der Landeszentrale für private Rundfunkveranstalter insgesamt einen Vertreter haben dürfen, auf den sie sich einigen müssen (Art. 26, Abs. 1,4). Die Kirchen gehören außerdem zu denjenigen Körperschaften, die eine Sendelizenz beantragen können. Schließlich wird ihnen weitgehend ein „Drittsenderecht“ eingeräumt (Art. 21, Abs. 1). Hinzu kommt, daß das Recht von Veranstaltern, Kurzberichte machen zu können („Öffentlichkeitspflicht") nach Art. 16, Abs. 3 nicht auf Kirchen und deren Einrichtungen angewendet werden darf, womit den Kirchen praktisch eine Art ,Abwehrrecht ${ }^{*}$ zugestanden wird. Ähnliches findet sich auch in Art. 1, Abs. 3 des schleswigholsteinischen Landesmediengesetzes.

\section{Saarland:}

Im Saarland gibt es derzeit nur einen einzigen privaten Hörfunksender, und der befindet sich zu einem Teil im Besitz des Saarländischen Rundfunks: „Radio Salü“ in Saarbrücken. Auch bei Radio Salü arbeitete zunächst (für die Diözese Trier) ein im Sold des PaulinusVerlages tätiger Redakteur, der ebenso wie bei den RPR-Studios nicht nur Kirchenfunk produziert, sondern in den übrigen Redaktionsablauf des Senders integiert ist. Derzeit ist diese Stelle allerdings nicht besetzt

\section{Schleswig-Holstein:}

Bereits im Referentenentwurf für das Rundfunkgesetz für Schleswig-Holstein vom 17.11.1983 war unter Art. 7 u. a. notiert, daß ,juristischen Personen des öffentlichen Rechts" keine Genehmigung für die Veranstaltung von Rundfunk erteilt werden könne "mit Ausnahme der Kirchen und der anderen öffentlich-rechtlichen Religionsgemeinschaften im Sinne von Art. $140 \mathrm{GG}^{\star}$. Diejenigen Lizenzbewerber haben Vorrang, die „alle bedeutsamen politischen, weltanschaulichen und gesellschaftlichen Gruppen zu Wort kommen“ lassen. Art. 21, Abs. 2 enthält das Drittsenderecht für die Kirchen.

Zwei Monate nach dem Sendestart des ersten Privathörfunksenders in Schleswig-Holstein, „Radio RSH“, begann im August 1986 die „Katholische Medienarbeit Schleswig-Holstein" in Kiel mit der Produktion von kirchlichen Beiträ- 
gen. Diese kleine katholische Redaktion arbeitet mit dem „Evangelischen Rundfunkdienst Nord" zusammen (einer entsprechenden Einrichtung der evangelisch-lutherischen nordelbischen Landeskirche), der mit Radio RSH lange vor dessen Sendestart eine Vereinbarung über die Zulieferung von kirchlichen Beiträgen getroffen hatte. Dort gab es auch einen sinngemäßen Zusatz, wonach die evangelische Kirche sich um eine Abstimmung mit der katholischen Kirche bemühen werde. Die beiden Kirchen vereinbarten einen Zulieferungsproporz von 4:1 (ev/rk), tatsächlich liegt der Proporz etwa bei 3:1. Die „ökumenische Redaktion“ ist auch räumlich zusammengefaßt, wobei die Studiotechnik und die übrige Ausrüstung gemeinsam genutzt werden.

Weil Radio RSH mittlerweile nicht mehr nur in Schleswig-Holstein gehört wird, Studios unterhält und Geschäfte macht, sondern auch in Mecklenburg-Vorpommern, heißt auch die Katholische Kirchenfunkredaktion jetzt, entsprechend ihrem evangelischen Pendant, „Katholische Medienarbeit Nord“.

Die Kirchenfunkredakteure beider Konfessionen sind in die RSH-Redaktion integriert. Seit Januar 1992 ist nicht mehr das Bistum Osnabrück direkter Träger der katholischen Redaktion in Kiel, sondern die Bistumszeitung „Kirchenbote Osnabrück“. Für Radio RSH wird neben den täglichen Verkündigungssendungen „Und nu kummst du ...", einer Erfindung des evangelischen Rundfunkdienst-Nord-Leiters Pastor Rainer Thun, vor allem am Sonntagmorgen ein Sonntagmorgenmagazin produziert, unter dem Namen „RSH-Sünndag“. Ein Sonntagmorgenmagazin ist mittlerweile auch beim konkurrierenden zweiten Privathörfunkveranstalter in Schleswig-Holstein „Delta-Radio“ in Kiel eingerichtet, zu exakt derselben Sendezeit, aber unter dem Namen „Delta-Radio himmlisch“.

\section{Hamburg:}

Je ein Sitz in der Anstaltsversammlung der Hamburgischen Anstalt für neue Medien (HAM) steht den Kirchen laut Landesrundfunkgesetz der Freien und Hansestadt Hamburg zu. Die Kirchen dürfen (ausnahmsweise, wie in vielen anderen Landesmediengesetzen) als Körperschaften des öffentlichen Rechts Rundfunkveranstalter sein.

Auch in Hamburg war wie in Schleswig-Holstein die evangelisch-lutherische Kirche mit den Privatradios im Geschäft, was auch dem Mitgliederverhältnis entspricht. Sie handelte mit den Veranstaltern kirchliche Sendezeiten aus, und ist deshalb auch federführend für die Kirchenfunkbeiträge. Auch dort kam jener Passus in die Verträge, daß die Belange der katholischen Kirche mit berücksichtigt werden sollten. Der „Evangelische Rundfunkdienst Nord“ nahm mit seiner Hamburger Redaktion im Jahr 1987 seine Tätigkeit auf. Bei den Hamburger Privatradios ließen sich für die Katholiken nur sehr wenige Beiträge unterbringen: $22 \mathrm{im} \mathrm{Jahr}$ 1991, 39 im Jahr 1992, die Mehrzahl davon bei „Radio Hamburg“. „Alster Radio“ nahm in zwei Jahren insgesamt vier Beiträge ab. Eine außerhalb der ökumenischvertraglichen Kooperation laufende Zuliefermöglichkeit tat sich bei ,Jazz Welle plus" auf, als dort 1992 jeweils am Donnerstagabend ein einstündiges monothematisches (Kirchen-)Magazin untergebracht werden konnte, mit einem Wortanteil von außergewöhnlichen $50 \%$. 


\section{Hessen: „Integriertes“ ökumenisches Schema}

In Hessen gehören die Kirchen zu jenen Institutionen, die laut Hessischem Landesgesetz über den privaten Rundfunk (HPRG) vom 30.11.1988 Rundfunk veranstalten können (Art. 5, Abs. 2, Satz 1). Bei beschränkter Übertragungskapazität haben diejenigen Antragsteller Vorrang, die entweder anbietungswillige Dritte aufzunehmen bereit sind (Art. 8, Abs. 2) oder erwarten lassen, daß sie die Ereignisse "des politischen, wirtschaftlichen, sozialen und kulturellen Lebens in Hessen" sachgerecht darzustellen in der Lage sind (Art. 8, Abs. 3). Auch im hessischen Privatrundfunkgesetz ist ein Drittsenderecht vorgesehen: Laut Art. 24 müssen der evangelischen und der katholischen Kirche sowie den jüdischen Gemeinschaften auf Wunsch angemessene Sendezeiten zur Verfügung gestellt werden; die Veranstalter können Selbstkostenerstattung verlangen. In der Anstaltsversammlung, der obersten Aufsichtsbehörde für den privaten Rundfunk in Hessen, sind die beiden Kirchen und der Landesverband der jüdischen Gemeinden in Hessen mit je einem Sitz vertreten (Art. 39, Abs. 1,3-5).

Die Kirchen waren in Hessen von Anfang an direkte Vertragspartner mit dem am 16.11.1989 den Sendebetrieb startenden Privathörfunkveranstalter „Radio FFH“. Am 26.01.1990 unterzeichneten die Diözesen Mainz, Limburg und Fulda gemeinsam mit den beiden evangelischen Landeskirchen Kurhessen-Waldeck und Hessen-Nassau mit Radio FFH einen auf vorläufig zwei Jahre befristeten Vertrag über kirchliche Sendezeiten. Vertragsgemäß gibt es täglich außer sonntags einen journalistischen Beitrag „Zwischentöne“ von jeweils zweieinhalb Minuten Länge. Es wird nicht wöchentlich zwischen katholischer und evangelischer Redaktion abgewechselt, sondern nach einer "integrierten“ Arbeitsweise. Ökumenisch ist auch das Sonntagmorgenmagazin „Kreuz \& Quer“, das ursprünglich auf den Dienstagabend plaziert war (zunächst auf vertragsloser Basis), das dann um eine Stunde ausgedehnt werden sollte, schließlich auf insgesamt vier Stunden erweitert und auf den frühen Sonntagmorgen verlegt wurde. Eine ungünstige Sendezeit, allerdings haben die beiden letzten Stunden erfreuliche Zuhörerzahlen. Die Kirchenfunkleute moderieren diese Sendung selbst.

\section{Nordrbein-Westfalen: Spät, aber gut organisiert}

Das nordrhein-westfälische Landesrundfunkgesetz wurde sehr spät, erst im Dezember 1986, verabschiedet. Weil das sozialdemokratisch regierte bevölkerungsreichste Bundesland dem Privatfunkgedanken zunächst nur sehr zögerlich zugetan war und sich entsprechend Zeit ließ, hatten auch die Kirchen in Nordrhein-Westfalen reichlich viel Zeit. Noch Mitte Januar 1987 konnte etwa der Generalvikar des Erzbistums Köln, Norbert Feldhoff, feststellen, die Zeit sei noch nicht reif für eine entsprechende Entscheidung. Zu dieser Zeit gehörte in den meisten Bistümern anderer Bundesländer die Kirchenfunkpraxis im Privatrundfunk schon zum Alltag.

Vorteile hatte das lange Zuwarten auch für die Privatfunkpraxis der Kirchen in Nordrhein-Westfalen: Es konnte mit Bedacht geplant werden, und weil die Medienstrategen eine NRW-spezifische Konstruktion erdachten (nämlich nach einer hitzigen Auswahlphase einen einzigen Mantelprogrammproduzenten für alle geplanten 46 NRW-Lokalhörfunkstationen zu lizenzieren (Radio NRW) und außer- 
dem für jedes Verbreitungsgebiet nur einen einzigen Lokalsender zuzulassen), waren die Verhältnisse zwischen Rhein und Weser so klar wie kaum anderswo.

Es gab für die nordrhein-westfälischen Bistümer die üblichen Optionen für eine Mitwirkung im zukünftigen NRW-Privathörfunk. Es sollte sowohl sog. „Verkündigungssendungen" als auch journalistische Beiträge aus dem kirchlichen Bereich geben. Die Zulieferung für das Mantelprogramm sollte durch eine kirchlichen Zentralredaktion in Essen geschehen. Daneben sollte aber auch jedes Lokalradio seine eigenen kirchlichen Beiträge bekommen, über deren Organisation in den Diözesen und vor Ort unterschiedlich entschieden werden konnte. Als dritte Schiene gab es die Hilfestellung für die Radioproduktionen der sog. „15\%-Gruppen" (Bürgerfunk) im Rahmen von Art. 24, Abs. 4 des NRW-Landesrundfunkgesetzes.

Im Dezember 1986 wurde das NRW-Landesrundfunkgesetz (erste Fassung) verabschiedet. Über drei Jahre später, am 01.04.1990, ging das „Radio-NRW'-Mantelprogramm auf Sendung.

Im Frühjahr 1990 beschlossen die Generalvikare der fünf Diözesen in Nordrhein-Westfalen, die Tellux-Tochter „Allgemeine Programmgesellschaft (APG)" in Wiesbaden und Stuttgart mit dem Aufbau einer katholischen Kirchenfunkredaktion in Essen zu beauftragen. Das ursprünglich in NRW nicht angesiedelte Unternehmen APG sollte zunächst „Verkündigungssendungen“ redaktionell betreuen. Die APG war durch ihr Stuttgarter Tochterunternehmen „Katholische Kirche im Privatfunk (KiP)" bereits im Auftrag der Diözese Rottenburg-Stuttgart tätig und hatte somit Privathörfunkerfahrung. Sie hob im August 1990 in Essen ein neues Tochterunternehmen unter dem Namen „Redaktion KiP-NRW. Redaktion für die Katholische Kirche im privaten Hörfunk-Rahmenprogramm Nordrhein-Westfalen" aus der Taufe.

Für die Verkündigungssendungen sollten jeweils bis zu fünf Personen aus jeder der fünf NRW-Diözesen Texte schreiben und möglichst auch selbst sprechen. Verantwortlich für den Inhalt der Beiträge wurden Bistumsbeauftragte in jeder der fünf beteiligten Diözesen: Manfred Becker-Huberti für Köln (jetzt Claudia Mies), Bert Gruber für Aachen, Günter Graf für Münster, Ferdinand Schulte-Berge für Essen, und Rainer Stratmann für Paderhorn (nach dessen Tod zwischenzeitlich kommissarisch Prälat Franz Hochstein, jetzt Chrisoph Quasten, Leiter des Medienzentrums für das Erzbistum Paderborn).

Lange vor dem Start von Radio NRW und der ersten Lokalradios hatten die NRW-Diözesen „Radiowerkstätten" aufgemacht zur Schulung von freien Autoren für die künftigen Lokalradios und für die Tätigkeit im Rahmen der „15\%-Bürgerfunkgruppen“. Die Kirche als Körperschaft darf, weil sie überall Mitglied der „Veranstaltergemeinschaft" der Lokalsender ist, die konkrete Radioarbeit für die Bürgergruppen nicht selbst leisten. Art. 24, Abs. 4, Satz 1 verbietet ihr auch selbst „15 $\%$ "-Sendezeit bei den Lokalradios zu beanspruchen. In der Praxis haben sich allerdings Sondermodelle herausgebildet, etwa in Köln. Dort teilen sich mehrere Radiowerkstätten die Bürgergruppen-Sendezeit bei „Radio Köln“ unter der Woche auf, und unter diesen ist auch eine katholische Gruppe. Sie hat am Dienstagmorgen 
„ihre“ Sendestunde in „Radio Köln“. In Gelsenkirchen haben sich sechs Radiofördervereine aus Gelsenkirchen und Essen, in denen auch kirchlich orientierte Leute sitzen, zur „Arbeitsgemeinschaft gemeinnütziger Radiovereine" im Bereich des Bistums Essen zusammengeschlossen. Im Erzbistum Paderborn gibt es „Radiorunden“ etwa unter dem fachlichen Rat der Radiowerkstatt Dortmund, deren Mitglieder im Rahmen der $15 \%$-Regelung Radiobeiträge machen.

Auf lokaler Ebene hat sich für die Kontaktbildung und -pflege zwischen Kirche und den Lokalradiostationen sowie für die Betreuung kirchlicher Themen die Einrichtung von kirchlichen "Radiokontaktern“ herausgebildet. Sie sollen vermitteln, beraten, anregen, auch selbst Beiträge machen. Im Erzbistum Köln etwa gibt es vier Kontakter. Im Bistum Münster (das im Rahmen des überdiözesanen Haushalts die Finanzbeiträge der fünf NRW-Diözesen für KiP-NRW in Essen zentral verwaltet) wurde nach längerem Zuwarten zunächst ein Redakteur für die Zulieferung von kirchlichen Themen für „Radio KW“ (Wesel) eingestellt. Er arbeitet in der Landjugendakademie Klausenhof, wo es seit Frühsommer 1992 das Produktionssstudio "Studio Klausenhof" gibt. Außerdem soll an drei regionalen Schwerpunkten im Bistum (Niederrhein, Westfalen und Ruhrgebiet) jeweils ein kirchlich angestellter Redakteur für Kirchenfunkbeiträge für die neuen Privatsender arbeiten.

Die nordrhein-westfälische Trennung zwischen Privatradio-Betriebsgesellschaft (BG), die das Geld und die Technik bereitstellt, und der Veranstaltergemeinschaft (VG), die das Programm kontrollierenund verantworten soll, hätte für die Kirchen in NRW keine Mitwirkungsrechte durch finanzielle Beteiligung erbracht. Laut NRW-Landesrundfunkgesetz steht ihnen aber jeweils ein Sitz (katholisch und evangelisch) in der Veranstaltergemeinschaft jedes Lokalradios zu. Diejenigen Dekanate, in deren Bereich ein Lokalfunk-Senderstandort geplant war, benannten Vertrauenspersonen, die dann vom Diözesanbischof zu kirchlichen Beauftragten in der Veranstaltergemeinschaft ernannt wurden. Inzwischen sitzt in jedem der bisher eingerichteten Kleingremien jeweils ein Vertreter der katholischen und der evangelischen Kirche. Eine entfernt vergleichbare Konstruktion ist im Bereich des privaten Hörfunks in Bayern zu finden, bei den dort anfangs gegründeten lokalen Kabelgesellschaften.

Das 1991 eröffnete neue Studio des Ruhrbistums Essen für die Produktion von Lokalfunkbeiträgen kann auch von der Redaktion KiP-NRW mitbenutzt werden. Ursprünglich war in Neuss produziert worden. Der erste Verkündigungsbeitrag „Augenblick mal" war am 03.09.1990 von Radio NRW an die Lokalstationen ausgestrahlt worden. Diese Beiträge (maximal zweieinhalb Minuten lang als eher informell daherkommende "tägliche Gedanken zur Zeit") werden wochenweise abwechselnd im Auftrag der katholischen und der evangelischen Kirche in NRW produziert. Seit 01.11.1990 strahlt Radio NRW auch das einstündige (journalistische) Sonntagsmagazin „Himmel und Erde" sonntagsmorgens aus. Auch hier wird bei der Produktion wochenweise zwischen der KiP-Redaktion und den Kirchenredaktionen der evangelischen Landeskirchen in NRW abgewechselt.

Radio NRW in Oberhausen gibt allerdings nicht nur den Lokalstationen die Musikfarbe für deren eigene lokale Sendestrecken detailliert vor, sondern auch den „Drittsendungs-Berechtigten“, also den Kirchen, die im Mantelprogramm ver- 
tragsgemäß in eigener Regie (und mit jeweils eigener Moderation) die „Himmel und Erde"-Sonntagssendung fahren. Diese Sonntagssendung im bevölkerungsreichen NRW hat mittlerweile neben dem Nachweis großer Hörerquoten wissenschaftliches Lob in einer Untersuchung erhalten, die im Auftrag der evangelischen Kirche gemacht wurde.

\section{Neue Bundesländer: Paragraphenroutine aus dem Westen}

Die Ausarbeitung der Landesrundfunkgesetze in den fünf neuen Bundesländern geschah zwar nicht genau zeitgleich, sie besaß aber ein Synchronelement durch die gleichermaßen vorhandene Notwendigkeit, auch die Belange des Rundfunks und damit auch des privaten Rundfunks zu regeln, und durch die routinierte Lobbytätigkeit insbesondere von Medienmultis, die für die schnelle Erschließung von Frequenzen (Hörfunk und Fernsehen) im Osten Deutschlands ihre Verbindungen spielen lassen wollten. Die konkrete Rundfunkgesetzgebung geschah dabei vorwiegend nach westdeutschen Ländervorbildern. In Berlin und Brandenburg gab es eine Besonderheit dadurch, daß für Privathörfunk bereits ein Westberliner Kabelgesetz bestand (einer der vier Kabelpilotversuche in der alten Bundesrepublik). Dessen Gremienrelikt, der Berliner Kabelrat, lebte weit über die Wiedervereinigung hinaus und mußte erst 1993 endgültig seine Kompetenzen an den Medienrat der Medienanstalt Berlin-Brandenburg (MABB) abgeben. Für die Kirchen ergaben sich in den neuen Bundesländern im Prinzip ähnlich lautende Festlegungen: Die Mitgliedschaft in den Versammlungen der Landesmedienanstalten wurde überall festgeschrieben. Im elfköpfigen „Landesrundfunkausschuß“ von Mecklenburg-Vorpommern haben die evangelische und die katholische Kirche sowie die jüdischen Gemeinden einen gemeinsamen Vertreter, über den sie sich abstimmen müssen, ansonsten überall einen jeweils eigenen Sitz. Das sog. Drittsenderecht (Sendezeiten für Gottesdienste und andere religiöse Sendungen) ist überall gewährleistet, überall ist Selbstkostenerstattung möglich. Hörfunk-Sendezeiten für Gottesdienstübertragungen wurden auch in den neuen Bundesländern bisher nicht in Anspruch genommen.

Die Kirchen sind als öffentlich-rechtliche Körperschaften überall in den neuen Bundesländern im Rahmen der für die Kirchen üblichen Ausnahmeregelung als mögliche Rundfunkveranstalter zugelassen, in Sachsen bei regionalen und landesweiten Vollprogrammen allerdings nur mit dem Status eines Zulieferers, in Sachsen-Anhalt dürfen sie selbst nur Vollprogramme veranstalten bzw. mitveranstalten.

In Thüringen verbietet das dortige Landesrundfunkgesetz den Mitveranstaltern von Privatrundfunk zugleich im Aufsichtsrat des jeweiligen Unternehmens zu sitzen.

In Sachsen und Mecklenburg-Vorpommern hat sich die Tellux-Tochter „Astra-Tel“ eingekauft, wenn auch nur mit sehr kleinen Anteilen: In MecklenburgVorpommern ist Astra-Tel mit $2 \%$ an „Antenne Mecklenburg-Vorpommern" beteiligt, in Sachsen mit 3,75 \% am Leipziger Privatsender „Radio PSR“. Die sächsische Kirchenbeteiligung wird zu 50 \% für die evangelische Kirche offengehalten. AstraTel und Radio PSR verhandelten eine wöchentliche Sendezeit von 30 Minuten für 
beide Kirchen zusammen aus: Eine tägliche Verkündigungssendung sowie eine wöchentliche Schwerpunktsendung. Die Kirchenfunkredakteure für Radio PSR sollen jeweils zur Hälfte von Radio PSR bzw. der jeweiligen kirchlichen Trägergesellschaft bezahlt werden.

„Radio PSR“ strahlt seit 01.12.1992 täglich an festen Sendeplätzen die Verkündigungssendung „Augenblick mal“ aus. Produzent ist wie in Baden-Württemberg und Nordrhein-Westfalen das Tellux-Tochterunternehmen „KiP“.

In Mecklenburg-Vorpommern ist der Aufbau einer Kirchenfunkredaktion derzeit im Gang, Sitz soll Schwerin sein. In Berlin gibt es beim Privatsender "r.s. 2" eine werktägliche Verkündigungssendung „Wort in den Tag“.

SUMMARY: The Church and private radio broadcasting. How do regional laws for broadcasting regulate promulgation of the Gospel and eclesiastical programmes in commercial broadcasting?

This text provides a stocking-taking of the actual legal regulations of the dual broadcasting system in Germany with regard to the question of the presence of the Church in private radio stations. With the introduction of commercial broadcasting the problem arrised, how - along with the public broadcasting institutions - the eclesiastical presence (capital investment of the Church, religious broadcasting, eclesiastical journalism) should find a legal regulation. The broadcasting treaty from 1987 granted explicitly an "appropriate" concideration of the Churches as ,relevant groups of the society" within the new private programmes. The author describes the often extremly varying conditions within the different ,Lands' of the Federal Republic of Germany. In this he gives an overview of the legal regulations and the actual presence of the Church or rather the eclesiastical programmes on the market of private radio broadcasting.

RÉSUMÉ: L'église sur le marché des radios privées. De quelle façon sont règlementés par le lois fédérales relatives à la radios les messages et les thèmes religieux dans les radios commerciales?

Le texte fournit un inventaire des règles légales en vigueur actuellement dans le système binaire de la radiodiffusion en Allemagne, tout en considérant la question à savoir quelle est la présence de l'église dans les programmes des responsables de radios privées. Avec l'apparition de la radio commerciale s'est posé le problème, pour les responsables de programmes privés, de savoir comment - à côté de la radio publique - la présence religieuse (parts de capitaux venant de l'église, émissions religieuses, journalisme religieux) devait être légalement règlée. Dans les nouveaux programmes privés, la convention de radiodiffusion de 1987 accorde expréssement aux religions en tant que "groupes sociaux signifatifs", une considération appropriée. L'auteur décrit les relations souvent très différentes d'un „Bundesland' à l'autre de la République fédérale et donne ainsi un aperçu en ce qui concerne les règlements légaux et la présence actuelle de l'église ou encore des émissions religieuses sur le marché des radios privées. 
RESUMEN: La Iglesia en el mercado de radio privado. Cómo se encuentran regulados el mensaje y los temas eclesiales en la radioemisión comercial por la ley de radiofonía de los etados federales?

El texto nos da una muestra de la reglamentación legal actual en el sistema de radiofonía dual de Alemania respecto a la pregunta sobre la presencia de la Iglesia en las radioemisoras privadas. Con el inicio de las emisoras comerciales se planteó el problema, cómo - junto a las radioemisoras públicas - se debería reglamentar jurídicamente la presencia de la iglesia (participación de capital de la iglesia,emisiones con temas eclesiales, periodismo eclesial) en las radios privadas. La ley de radiofoniá de 1987 concede expresamente a la Iglesia como "grupo social relevante“ un espacio „adecuado" en los nuevos programas privados. El autor describe la situación, a menudo muy diferente, en los diferentes estados federales y con ello da una visión de la reglamentación jurídica y la actual presencia de la Iglesia, o de las emisiones eclesiales en las radios privadas. 